New windows on massive stars: asteroseismology, interferometry, and spectropolarimetry

Proceedings IAU Symposium No. 30\%, 2014

G. Meynet, C. Georgy, J. H. Groh \& Ph. Stee, eds.

(C) International Astronomical Union 2015 doi:10.1017/S1743921314006395

\title{
Evolution of the rotational properties and nitrogen surface abundances of B-Type stellar populations
}

\author{
A. Granada ${ }^{1}$, G. Meynet ${ }^{1}$, S. Ekström ${ }^{1}$, C. Georgy ${ }^{2}$ and \\ L. Haemmerlé ${ }^{1}$ \\ ${ }^{1}$ Geneva Observatory, University of Geneva, Maillettes 51, 1290 Sauverny, Switzerland \\ email: anahi.granada@unige.ch \\ ${ }^{2}$ Astrophysics group, EPSAM, Keele University, Lennard-Jones Labs, Keele, ST5 5BG, UK

\begin{abstract}
Stellar evolution models predict that rotation induces the mixing of chemical species, with the subsequent surface abundance anomalies relative to single non-rotating models, even during the main sequence (MS) evolution. The lack of measurable nitrogen surface enrichment in MS rotating stars, such as Be stars, has been interpreted as being in conflict with evolutionary models (e.g. Lennon et al. 2005; Hunter et al. 2008). In order to have an insight on the kind of ambient we do or we do not expect to find enriched rotating stars, we use our new population synthesis code, to produce synthetic intermediate-mass stellar populations fully accounting for stellar rotation effects, and study their evolution in time.
\end{abstract}

Keywords. stars: evolution, stars: rotation, stars: abundances.

\section{Introduction}

We use SYCLIST (SYnthetic CLusters Isochrones \& Stellar Tracks, Georgy et al. 2014) to study how the fraction of single rotating and nitrogen-enriched B-Type stars evolve, using as inputs the rotating stellar models from Georgy et al. (2013) and Granada \& Haemmerlé (submitted). We define as B-Type stars those objects with $\mathrm{T}_{\text {eff }}$ between 10000 and $30000 \mathrm{~K}$. Early B-type have $\mathrm{T}_{\text {eff }}>19000 \mathrm{~K}$. We consider a star as nitrogenenriched if $(\mathrm{N} / \mathrm{H}) /(\mathrm{N} / \mathrm{H})_{\text {ini }}>2$. Apart from coeval populations, we also computed the fraction of rotating and enriched stars for continuous star formation.

\section{Results}

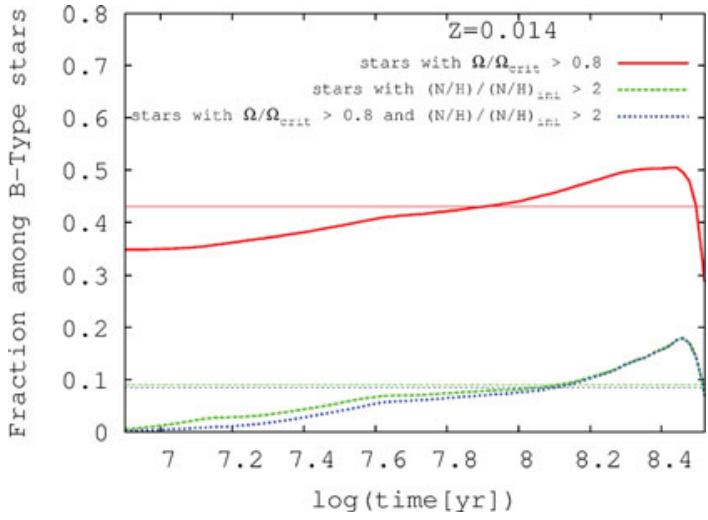

Figure 1. Evolution of different types of B star.
In Fig. 1, the thick lines represent the evolution of the fraction of B-type stars at $\mathrm{Z}=0.014$ with $\Omega / \Omega_{\text {crit }}>0.8$ (solid red), of objects with $(\mathrm{N} / \mathrm{H}) /(\mathrm{N} / \mathrm{H})_{\text {ini }}>2($ dashed green), and with $\Omega / \Omega_{\text {crit }}>0.8$ and $(\mathrm{N} / \mathrm{H}) /(\mathrm{N} / \mathrm{H})_{\text {ini }}>2$ (dotted blue). The thin lines indicate the same values, for continuous star formation. The values obtained for an age of $25 \mathrm{Myr}$ are listed in column 6 of Table 1. 
Table 1. Fraction of B and of early-B stars, having properties described in column 1.

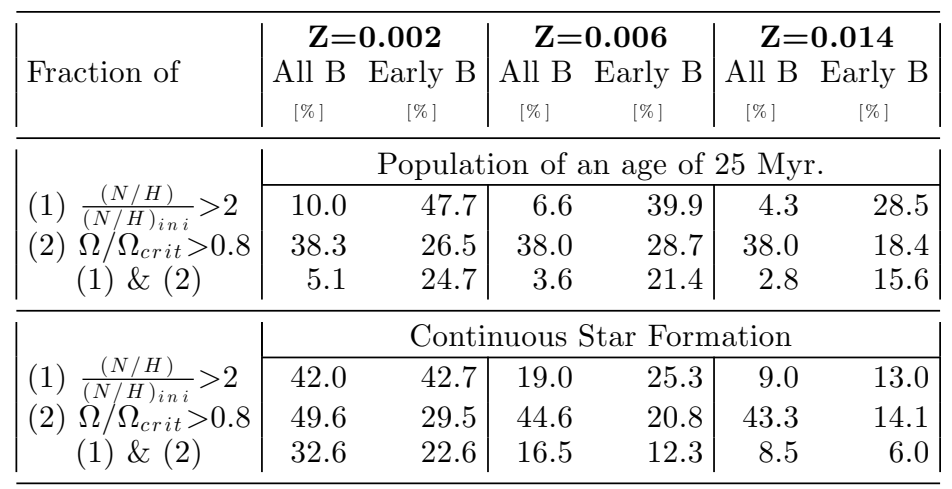

Fig. 2 shows the Colour Magnitude Diagram (CMD) of the MS of a synthetic cluster of an age of 25Myr with 100 intermediate mass stars, produced by SYCLIST. Black circles indicate late B stars, red squares indicate early-type B stars. Solid symbols indicate rapidly rotating stars and the blue romboid ${ }^{\mathrm{s}}-$ those objects with a large nitrogen enrichment. As expected for this age, around $2 \%$ of the $\mathrm{B}$ stars are rapidly rotating and nitrogen enriched. Because we can account for inclination effects to build our synthetic clusters, in the example given, the brightest blue square has $\mathrm{V} \operatorname{sini}=117 \mathrm{~km} / \mathrm{s}$ and the other $\mathrm{V}$ sini $=400 \mathrm{~km} / \mathrm{s}$. In terms of the surface velocity, only one of this objects would seem to be rapidly rotating and enriched.

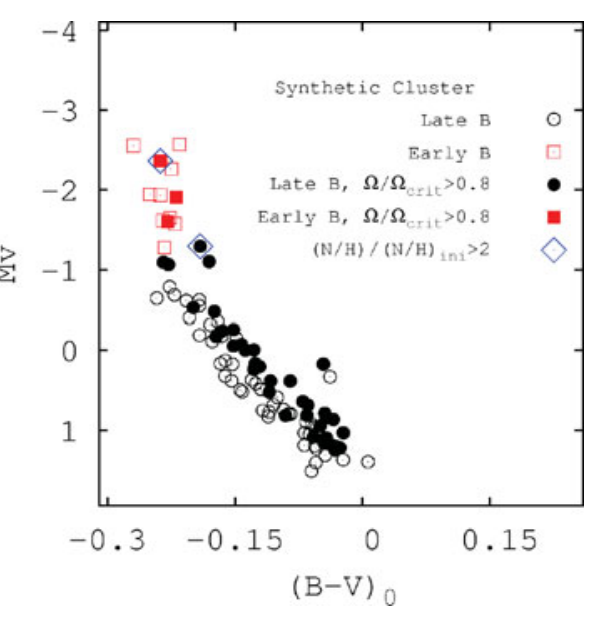

Figure 2. Synthetic CMD, 25 Myr old.

\section{Conclusions}

- We expect a very low fraction of enriched rotating B-type stars in coeval young populations.

- These fractions are larger among early B-type stars and at smaller Z.

- When continuous star formation is considered, the fractions of rapidly rotating and enriched stars among B-type stars can differ significantly from coeval populations. At $\mathrm{Z}=0.014$, the fraction is larger among all B stars, but is reduced among early-B stars.

- All these results are dependent on the initial velocity distribution, on the definition of rapidly-rotating and of what we call an enriched stars. We are currently exploring all these dependences.

\section{References}

Georgy, C., Ekström, S., Granada, A., et al. 2013, A\&SA 553, A24

Georgy, C., Granada, A., Ekström, S., et al. 2014, A\&A 566, A21

Hunter, I., Brott, I., Lennon, D. J., et al. 2008, ApJ (Letters) 676, L29

Lennon, D. J., Lee, J.-K., Dufton, P. L., \& Ryans, R. S. I. 2005, A 64 A 438, 265 